\title{
Use of Ultrasound in Rape Oil Extraction Process
}

\author{
Hrynchuk Y. ${ }^{1}$, Reutskyy V. ${ }^{1}$, Matsipura P. ${ }^{1}$ \\ 1. Department of Physical, Analytical and General Chemistry,National University "Lviv Polytechnic", Ukraine, \\ Lviv, Bandera street 12, E-mail: yurii.m.hrynchuk@lpnu.ua
}

\begin{abstract}
An extraction method for the production of vegetable oils is essential in the food industry. Getting oil in this way is the most economical, provides maximum degreasing of oilseeds, and allows you to achieve high quality of the product. The main advantage of the extraction method of obtaining vegetable oils in comparison with the press is the significant increase in the yield of oil, even in the processing of low-oil seeds.
\end{abstract}

Keywords - asphalt concrete, modifier, epoxide, bitumen, strength, water resistance, ultrasound.

\section{Introduction}

All extraction processes are limited by diffusion at the interface of the phases through a diffusion layer with gradient concentration of the extraction of matter. Traditional extraction methods are long-lasting. Ultrasound can significantly accelerate the extraction process, increase output, reduce the cost of extraction of matter and improve the quality of the product.

The influence of ultrasonic oscillations with $22 \mathrm{kHz}$ frequency, low resonance cavitation $(17-100 \mathrm{~Hz})$ and vibration turbulent devices on the output, composition of extraction products was investigated and analyzed. It has been shown that in all cases the yield of the extract increases, but the quantitative characteristics of the product obtained are different.

The process of epoxidation of obtained extracts and their application in the process of modification of road bitumen was investigated. Taking into acount the considerable time of the modification process, and some differences in the results obtained in studies using oils obtained by different extraction methods, a decision was made on the need to intensify the processes of modification of road bitumen by use of ultrasound [1].

Different methods of extraction, their advantages and disadvantages were considered. Some conclusions are made about the use of ultrasound in the extraction process for the production of oils, and in the process of modifying of road bitumen with epoxy compounds.

\section{Conclusion}

The use of cavitation in the process of modifying of road bitumen for reducing of the modification time, comparative analysis of the properties of bitumen, modified using cavitation and without it, as well as the study of the effect of these changes on the properties of asphalt concrete were made. The industrial use of the obtained, high-quality and cheap vegetable-based modifier will promote the expansion of the use of BMP and, consequently, increase the durability of road coverings.

\section{References}

[1] Mykhailo Nykypanchuk, Yurii Hrynchuk and Mykola Olchovyk, "Effect of Modified Bitumen on Physico-mechanical Properties of Asphalt Concrete”, Chemistry and Chemical Technology, Vol.7., № 4, pp. 467-470, 2013. 